\title{
Chromatic Mach bands: Behavioral evidence for lateral inhibition in human color vision
}

\author{
COLIN WARE \\ University of New Brunswick, Fredericton, New Brunswick, Canada \\ and \\ WILLIAM B. COWAN \\ National Research Council of Canada, Ottawa, Ontario, Canada
}

\begin{abstract}
Apparent hue shifts consistent with lateral inhibition in color mechanisms were measured, in five purely chromatic gradients, for 7 observers. Apparent lightness shifts were measured in achromatic versions of the same gradients, and a correlation was found to exist between the magnitude of the chromatic shifts and the magnitude of the achromatic shifts. The data can be modeled by a function that approximates the activity of color-sensitive neurons found in the monkey cortex. Individual differences and the failure of some previous researchers to find the effect can be accounted for by differences in the function parameters for different observers.
\end{abstract}

One of the intensity gradients used by Ernst Mach $(1886 / 1967)$ to demonstrate what have become known as "Mach bands" is graphed in Figure 1 (curve C). Observers of a pattern with this luminance profile report a bright band at the point where the luminance begins to decline. As with others like it, this observation represents one of the principal sources of evidence for spatially inhibitory interactions in the human visual system.

If, instead of varying in luminance from light to dark, the gradient was chromatic, running, for example, from red to green, would a chromatic Mach band appear? The answer is controversial. Some experimenters who have looked for chromatic Mach bands have found them (Daw, 1964; Jacobson \& MacKinnon, 1969); others have not (Ercoles-Guzzoni \& Fiorentini, 1958; Fry, 1948; Green \& Fast, 1971; Thouless, 1922; Van der Horst \& Bouman, 1967).

The well-established phenomena of chromatic contrast make the dispute over chromatic Mach bands puzzling, since the same mechanisms may be expected to produce both sets of phenomena. Experimenters who find no chromatic Mach bands sometimes question the evidence of those who do, on the grounds that inadequate measures were taken to ensure that the chromatic gradient had no achromatic component. If the chromatic gradient did have

This research was carried out at the National Research Council of Canada while the first author was employed as a Research Associate at that institution. We wish to thank P. K. Kaiser, C. R. Ingling, and G. Wyszecki for advice concerning appropriate ways to equate luminance in heterochromatic patterns. Also, we wish to thank the many visitors to our laboratory who looked at the displays and spurred on our progress with their interest. Lastly we wish to thank an anonymous reviewer whose comments concerning Experiment 3 enabled us to strengthen the presentation of that experiment.

C. Ware's present address is: School of Computer Science, Box 4400, Fredericton, N.B., Canada E3B 5A3. W. B. Cowan's mailing address is: Optics Section, Division of Physics, N.R.C. Canada, Ottawa, Ont., Canada K1A 0R6. an achromatic component, chromatic shifts could be caused by a coupling to the achromatic effect through, for example, the Bezold-Brücke effect. They also argue that, because prolonged viewing was allowed, chromatic Mach bands may be an artifact of successive contrast and eye movements and not evidence for lateral interactions. On the other side, those who find chromatic Mach bands can point out that spurious negative results will occur if the effect varies widely in strength among patterns and observers, especially since most studies used small numbers of patterns and observers.

Physiological evidence is similarly equivocal. Colorsensitive cells in the retina and lateral geniculate nucleus of the monkey do not have the right characteristics to produce chromatic Mach bands (Ingling \& Drum, 1973), but the double opponent cells reported in the monkey visual cortex do (Michael, 1978). Thus, from several perspectives, the possible existence of chromatic Mach bands and their relationship to lateral interactions is important to our understanding of how humans process color information. The present series of experiments addresses these issues, seeking conclusive psychophysical evidence for the phenomenon of chromatic Mach bands.

\section{GENERAL METHOD AND STIMULI}

We studied five different gradients in chromatic and achromatic versions (see Figure 1). All of these patterns show contrast effects (i.e., the enhancement of differences) when achromatic. However, some are of a type usually used in the study of "simultaneous contrast" phenomena; others produce "Mach band" effects. The gradients were produced by spinning, at $60 \mathrm{~Hz}$, disks to which patterns of paper were attached. Different patterns gave the five gradients shown in Figure 1 as well as the inverses of each (colors reversed or black and white reversed). Fixation guides were used; these consisted of small white marks located beneath the test pattern at the arrowed locations shown in Figure 1. The matching field was a spinning variable-sector disk, its sectors covered with the 


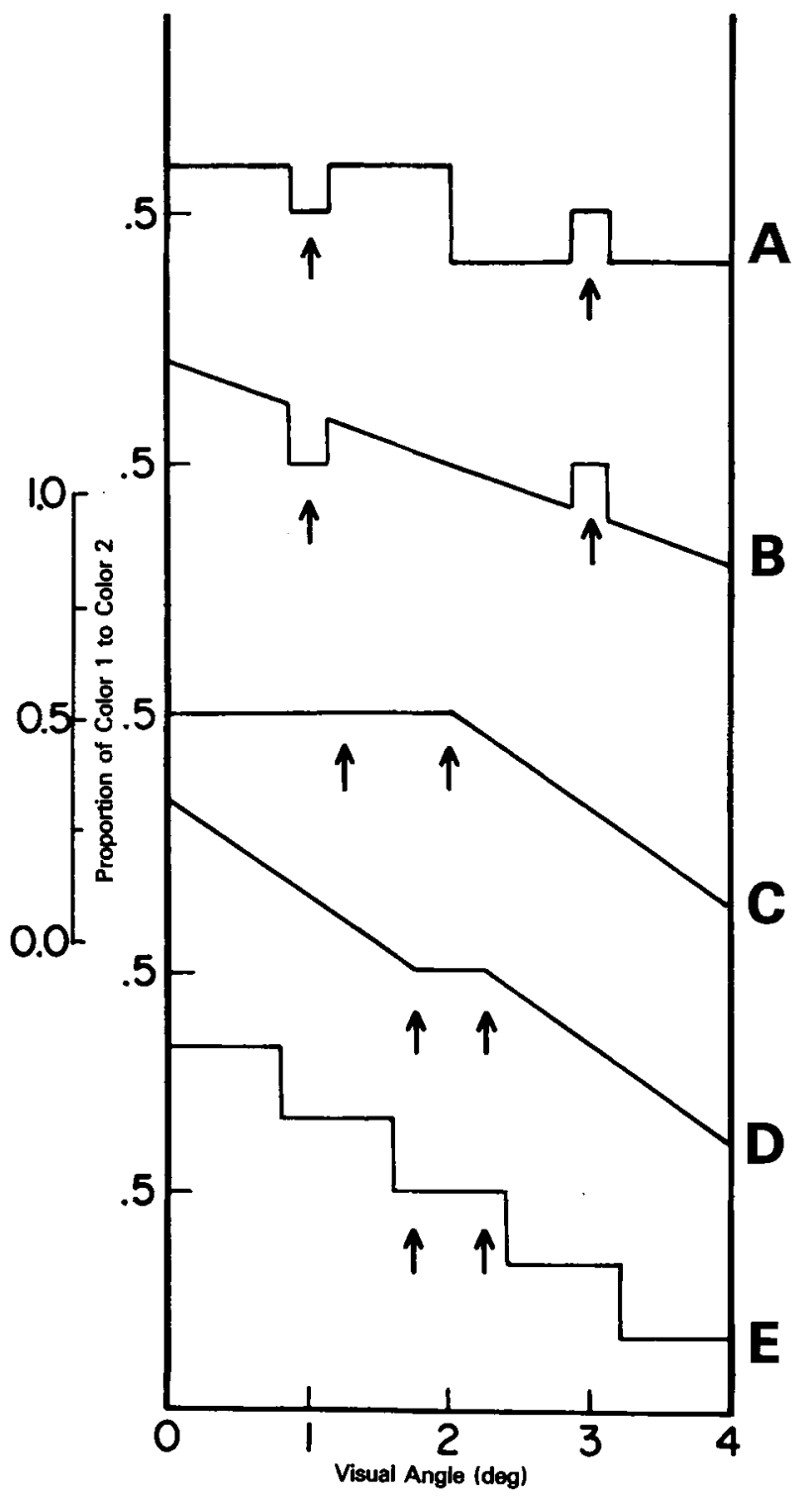

This pattern has the form of the standard demonstration of simultaneous brightness of color contrast. A region on a bright (or colored) background is compared to a region on a dark (or differentty colored) background. For some earty versions see Chevreul (1839).

A variation on ' A'. Since there is evidence that edges play a considerable part in brightness perception, it is of interest to remove the abrupt edge between the beckgrounds in ' $A$ ' while retaining the seme overall contrast with the test regions.

Emst Mach (1886) first discovered his famous contour enhancement effect using a luminance gradient like this. A bright band is seen at the central arrow. Previous researchers failed to find chromatic Mach bands in chromatic versions of this gradient.

This pattern was used by $O^{\prime} B$ rien (1958) to investigate the Mach band effect. It has the useful property of increasing the size of the effect over that which is seen in gradient ' $\mathrm{C}$ '.

Ranked steps of uniform luminance are seen as non uniform by most observers. This offect has often been linked to Mach bands and it has been found to occur in chromatic versions of this gradient. Chevreul (1839) is credited with discovering the effect.

Figure 1. Graphs showing the five gradients used in the experiments. The arrows show the points to which matches were made. The scale on the ordinate denotes the proportion $(p)$ of one of the two component colors in the gradient at a particular point on the gradient. The amount of the other component is $1-p$. Notes summarize historical antecedents and theoretical significance of each pattern.

same colored papers as the disks used for the stimulus gradients. A micrometer drive that was attached to the matching disk allowed subjects to vary the relative sizes of the red and green sectors and, thus, the color mixture. In this way, an exact physical match could be obtained with any part of the stimulus gradient. Sections of the gradient disk and the matching disk were masked off to create the display field shown in Figure 2. The subjects observed the display through a large beam splitter set at $45^{\circ}$ to the line of sight. By alternately illuminating the chromatic display and a neutral white display of the same luminance, shape, and position, we were able to study the effects of intermittent exposure. The layout of the apparatus is diagramed in Figure 3.

\section{Chromatic and Achromatic Gradients}

Each stimulus gradient consisted of linear mixtures of the endpoint colors. The proportion of each endpoint color present at a given portion of the gradient is shown in Figure 1. The endpoint colors were defined by the colors of the papers used to make the disks and the colors of the filters through which they were illuminated. Three different sets of the five gradients shown in Figure 1 were used as stimuli. The sets were designed to maximally stimulate the red-green opponent channel, the yellow-blue opponent channel, and the achromatic channel, respectively. To create gradients that selectively affected the opponent channels in this way, it is necessary that the two colors defining the endpoints of a gradient differ 


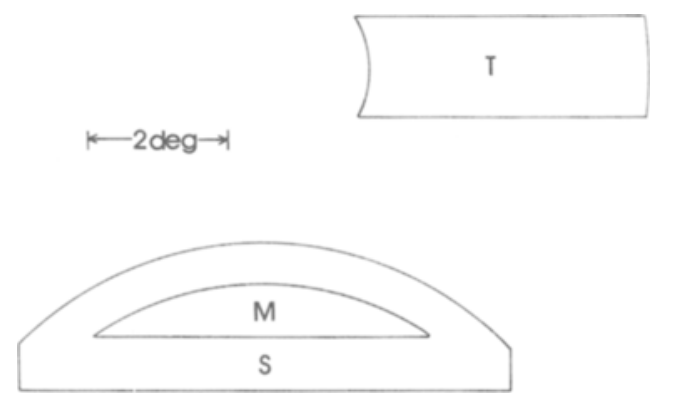

Figure 2. Scale drawing of the masked display as seen by the observer. The almost rectangular area (T) contained the chromatic or achromatic gradient. The chord-shaped region contained the matching field (M), with a neutral surround (S), which became the color of the illuminant.

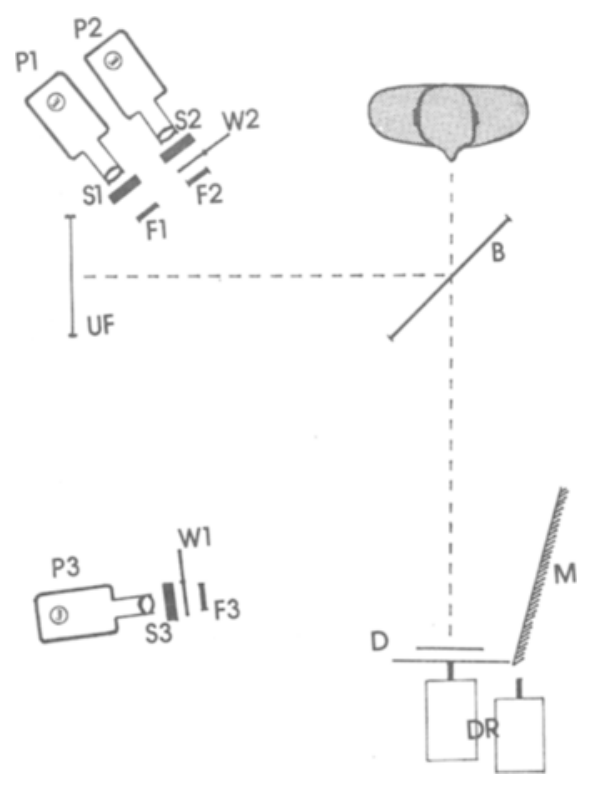

Figure 3. Diagram showing the layout of the major components of the apparatus. The mirror (M) was placed to distribute the light from projectors (P1 and P2) in such a way as to create a more uniform illumination over the test and matching disks (D). The light from another projector (P3) was also reflected from the same mirror to illuminate the uniform neutral field (UF). The stimuli were viewed through a large beam splitter (B), which meant that either the uniform field was seen or the test and matching disks were seen, depending on what was illuminated at a particular instant. Shutters (S1, S2, and S3) were used to control the timing of the illumination of the test and matching disks and the uniform feld. Filters (F1, F2, and F3) and neutral density wedges (W1 and W2) were used to control the illumination of the fields. Also shown is the observer (O) and the drive motors (DR) used to spin the disks.

only on the channel to be stimulated. The following set of endpoints were created after much experimentation with combinations of colored papers and illuminating lights. Table 1 shows the three sets of endpoint colors in CIE tristimulus values.

\section{Red-Green Gradients}

To obtain the red-green chromatic gradients, we made the disks from red and green papers and illuminated them by red and green sources of variable intensity. The sources were adjusted by the method of flicker photometry so that red and green areas, and hence all intermediate areas, were of equal luminance for each subject.

The CIE chromaticity coordinates of the two components of the chromatic gradients can be given only if it is assumed that the subjects are equating the luminance at the same balance that the 1924 CIE V $(\lambda)$ does. The chromaticity coordinates given for the red-green gradients assume this.

\section{Yellow-Blue Gradients}

Yellow and blue papers illuminated through yellow and blue filters were used for the yellow-blue gradients. Since changing the relative intensity of the two sources drastically changed the chromaticity of the blue paper used for the disk, and since collinearity with the tritanopic copunctal point is necessary to maximally excite the yellow-blue channel and not excite the red-green channel, the flicker method proved impractical for making these gradients equiluminous. Thus, it was not possible to individually set the relative luminances. We therefore made the stimuli equiluminous by using a photometer that embodied a good $V(\lambda)$ approximation.

\section{Achromatic Gradients}

The achromatic gradients were made of black and white papers. These were illuminated by light from projectors that was filtered to approximate CIE standard illuminant D65.

\section{Metric for Describing Gradients}

To describe the patterns and express the results, we constructed a scale that gave the value of unity to the distance between the two colors (1 and 2). The tristimulus values of scale value $p$ $(0 \geq p \geq 1)$ are $X(p)=p X_{1}+(1-p) X_{2}, Y(p)=Y_{1}+(1-p) Y_{2}$, $Z(p)=p Z_{1}+(1-p) Z_{2}$. Chromaticity coordinates can be obtained, for any given part of the stimulus gradient, by the formulas $x=X /(X+Y+Z)$ and $y=Y(X+Y+Z)$.

\section{EXPERIMENT 1}

The purpose of the first experiment was to determine the magnitude of the simultaneous contrast effect for each of the five gradients shown in Figure 1 and to compare those effects for chromatic and achromatic versions of the patterns.

\section{Method}

Subjects. Seven subjects were used. All were color normals, as measured by the Farnsworth-Munsell 100 Hue Test and H-R-R pseudoisochromatic plates. Also, all subjects had had some previous experience with psychophysical experiments involving color, although only two, C.W. and W.B.C., were familiar with relevant theories of color perception.

Procedure. To assess the effect for a given gradient, we had the subject first make six matches to one of the points arrowed in Figure 1 and then six matches to the other point. The measured size of the effect was the difference between the two sets of matches.

Table 1

Endpoint Colors in CIE Tristimulus Values

\begin{tabular}{lrrrrrr}
\hline & \multicolumn{6}{c}{ Stimulus Component Colors } \\
\cline { 2 - 7 } & \multicolumn{1}{c}{$\boldsymbol{X}$} & \multicolumn{1}{c}{$\boldsymbol{Y}$} & $\boldsymbol{Z}$ & \multicolumn{1}{c}{$\boldsymbol{X}$} & \multicolumn{1}{c}{$\boldsymbol{Y}$} & \multicolumn{1}{c}{$\boldsymbol{Z}$} \\
\hline Red-Green & 24.97 & 11.75 & 0.01 & 7.37 & 11.75 & 0.80 \\
Yellow-Blue & $\mathbf{6 . 1 3}$ & $\mathbf{6 . 4 0}$ & $\mathbf{0 . 8 0}$ & 7.72 & 6.40 & 7.94 \\
Black-White & 5.97 & 5.80 & 5.80 & 36.27 & 35.20 & 35.20 \\
\hline
\end{tabular}



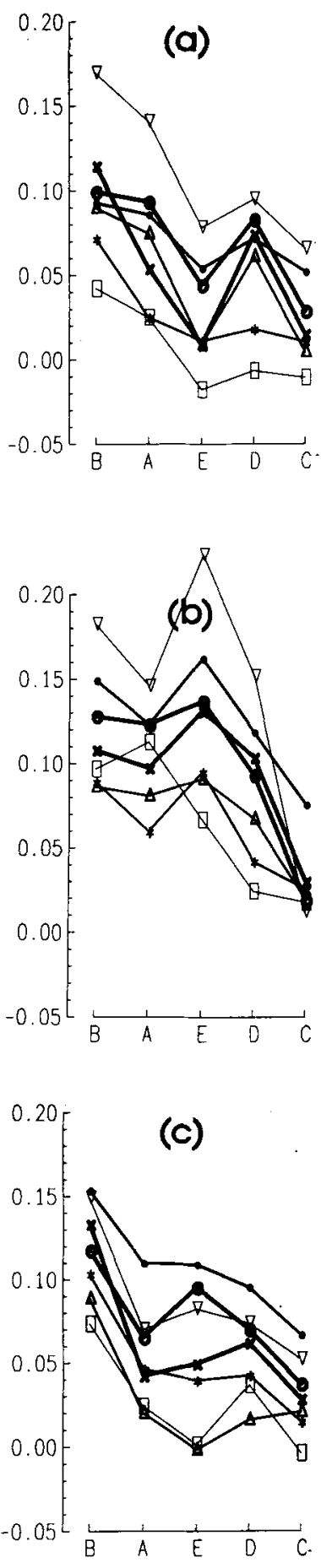

Figure 4. Graph showing the measured contrast effects under continuous viewing conditions for 7 subjects viewing each of the five gradients. The results shown are those obtained using the red-green gradients, (a), the yellow-blue gradients (b), and the achromatic gradients (c). Each observer is represented by a particular symbol on all three graphs. The scale on the abscissan denotes the amount of the contrast effect as a proportion of the total distance in tristimulus space between the two component colors used to generate a particular gradient.
On a different day, the subject made a second set of matches to that gradient with the order of the points matched reversed. Also, since the chromatic inverse of each pattern was essentially a leftto-right reversal of the gradient, the estimate of the size of the effect for a given gradient was the average of the results of four sessions.

\section{Results and Discussion}

The results from Experiment 1 are summarized by the three graphs shown in Figure 4. These show the mean size of the contrast effect for each subject and each pattern, in each of the three color categories: red-green, yellow-blue, and achromatic. The size of the effect is given in units that are decimal fractions of the distance in tristimulus space between the two endpoint colors.

Notice the following features: (1) All patterns show contrast effects, chromatic contrast in the case of the chromatic gradients and achromatic contrast in the case of the achromatic gradients. For example, in the case of redgreen gradients, areas with green surrounds look redder than areas with red surrounds. (2) There is great intersubject variation; some subjects consistently produced matches indicating larger contrast effects. (3) Given the large individual variation, the magnitude of the effect is roughly comparable for the achromatic, red-green, and yellow-blue versions of the patterns. (4) There is great interpattern variation, some patterns consistently giving larger contrast effects than others. However, the patterns that produced the largest measured contrast effect for the chromatic gradients also produced the largest result for the achromatic gradients. In particular, there is no evidence that the Mach band gradients (C and D) were qualitatively different. Also, classical Mach band gradient C gave the smallest effect for all chromatic and achromatic versions.

There was only one pattern that showed different behavior, depending on the colors used to produce the gradient. Pattern E, the staircase pattern, showed rather striking differences depending on the color modality. Thus, individual differences were relatively larger with the achromatic versions of this pattern than with the chromatic versions. In the red-green version, the effect for this pattern was reduced relative to the effects for patterns $B$ and D, whereas in the achromatic pattern the size of the effect was comparable to that produced by those two other patterns. Finally, and most remarkably, in the yellow-blue version, this pattern produced, for most subjects, a larger effect than any other pattern.

The above evidence suggests that the same kind of mechanism mediates all the contrast effects (except in E), and that the mechanism is incorporated separately in the red-green, yellow-blue, and achromatic "channels."

If we ignore pattern $E$, the results, taken together, can be explained by lateral inhibition in chromatic and achromatic channels. The intersubject variation can be explained by the occurrence of different amounts of inhibition at the level in the visual system at which these effects take place. However, we did two control experiments to examine possible alternative explanations. 


\section{EXPERIMENT 2}

It has been suggested that apparent chromatic contrast might be caused by lateral inhibition in the achromatic channel, which in some way, not usually specified, pulls the chromatic appearance along with the chromatic effect. To exclude this possibility, the achromatic channel must be carefully nulled. Because the flicker photometric technique for nulling the achromatic channel might be inappropriate, or insufficiently accurate, we used Daw's (1964) procedure, allowing 4 subjects to vary the relative red-green luminances while looking at pattern D. Their instructions were to look for a minimum in the chromatic contrast, or its disappearance, while looking at the pattern and adjusting the luminance of the red component of the pattern through its entire range. None of the subjects reported the disappearance of the chromatic effect, except in the trivial case in which there was no red light and the gradient degenerated to a green luminance gradient. Neither did they report any diminishing of the effect as the gradients passed through the state in which the component hues appeared to be equiluminous.

\section{EXPERIMENT 3}

Some investigators think that chromatic contrast may be caused by small eye movements that cause differential adaptation near changes in the pattern (Wooten, 1970). Even though there exists no coherent model of Mach bands or other contrast phenomena based on eye movements, we nevertheless decided to try to establish whether or not eye movements were contributing to the effects we were observing.

Such eye movements cannot be eliminated by fixation instructions, but adaptation can be decreased by intermittent viewing. Prior experimentation had shown that, practically, 1-sec intermittent exposures were the shortest that could be used. The task had required that in this interval the subject fixate the relevant part of the test field and the matching field with an oblique saccade in between. At the most, two fixations to the test field were possible;

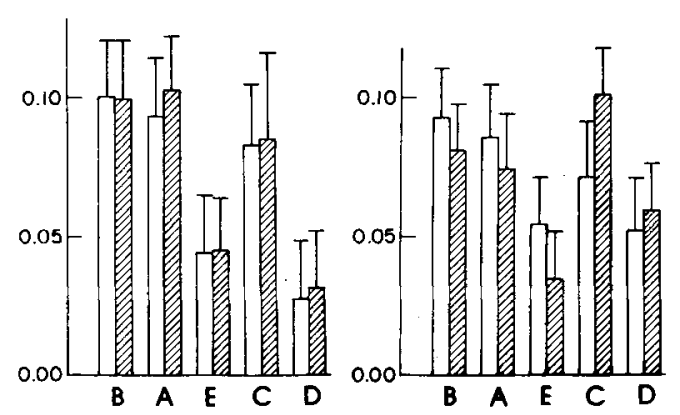

Figure 5. Histogram showing the results of Experiment 3. The data are for 2 subjects for all five gradients. Open bars show measured contrast effects under continuous viewing; hatched bars show the effect measured under intermittent viewing. At the top of each bar is a marker showing $1 S D$.

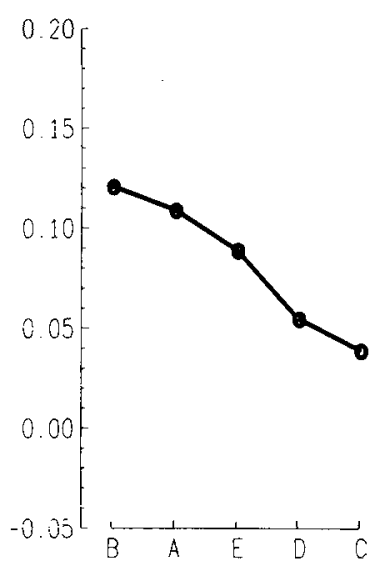

Figure 6. Graph showing the contrast effects predicted by a hypothetical double-opponent chromatically sensitive neuron for all five gradients. The same effects are predicted by an opponent achromatic neuron.

generally only a single fixation occurred. This should certainly be sufficient to drastically reduce eye-movementinduced edge effects.

Two subjects matched all five patterns under both continuous and intermittent viewing conditions ( $1 \mathrm{sec}$ of pattern, $4 \mathrm{sec}$ of neutral field). The results (Figure 5) show no diminution of the effect in intermittent viewing.

One-second exposures seem to be a practical limit, below which matching cannot be done. Nevertheless, we had 4 subjects perform a somewhat different task with shorter exposures (.5 sec of pattern, $2.5 \mathrm{sec}$ of neutral field). The subjects were asked to attempt to remove the chromatic Mach bands by directly manipulating the color balance. None could do so.

Although it is possible that substantial adaptation can occur in $.5 \mathrm{sec}$ and that adaptation after $1 \mathrm{sec}$ is the same as in continuous exposure, we consider this to be unlikely. We feel that the results described above, combined with the positive results of our lateral inhibition model (below), make the eye-movement explanation improbable.

\section{THE LATERAL INHIBITION MODEL}

To see how well lateral inhibition could account for the results, we constructed a model based on color-sensitive neurons found in the monkey cortex (Michael, 1978). These are concentric double-opponent neurons with receptive fields of two types: One has a circular center excited by red, inhibited by green, and an annular surround excited by green, inhibited by red; the second type of cell is the chromatic converse of the first. We created a hypothetical neuron of this type, with its center surround structure modeled by the difference of Gaussians function: $\exp \left(-x^{2} / \sigma^{2}\right)-w \cdot r \cdot \exp \left(-w^{2} x^{2} / \sigma^{2}\right)$, where $x$ is the distance from the center of the distribution in minutes of arc, $\sigma$ is the width of the center, $w$ is the ratio of center width to surround width, and $r$ is the ratio of inhibition to excitation (Wilson \& Bergen, 1979). Figure 6 shows the difference in response when such a cell-with a center 
half width of $10^{\prime}$ and a surround 10 times as large, parameters similar to those of monkey cortex-is positioned at the arrows of each pattern in Figure 1. The parameters were: $\sigma=6^{\prime}, w=0.1$, and $r=0.6$. Varying cell parameters (center and surround sizes, inhibition ratio) varies the size of the effect but not the rank order of the patterns. This is interesting, since human subjects give results of varying magnitude but of consistent order. Note that, with the exception of pattern $\mathrm{E}$, the model ranks the patterns in the same order as human subjects do.

We do not have a coherent explanation for the results obtained with pattern E. However, we suggest that pattern $E$ may give a small result because there is a visual mechanism that is not included in the model, but which "fills in" between edges and behaves differently for the different chromatic and achromatic channels. This idea is supported by our results with a chromatic Cornsweet pattern (Ware \& Cowan, 1983) for which the model gives no effect and humans give a negative one. The Cornsweet effect can also be predicted by a "filling-in" mechanism, and it gave different effects for the different chromatic and achromatic channels; however, the largest effect was obtained for the achromatic pattern and the smallest effect was obtained for the chromatic pattern. Clearly, the possible existence and operation of a "filling-in" mechanism is a profitable subject for future experimentation.

\section{CONCLUSION}

The finding that the same individual variation underlies the large simultaneous contrast effects and the relatively smaller Mach-band effects strongly suggests that these effects are subserved by the same mechanism. As for the identity of this mechanism, although eye movements are not absolutely ruled out, lateral inhibition is at present the strongest candidate. The interpattern and intersubject variability in our study also provides a reason why investigations of chromatic Mach bands have been so inconclusive. Most of the previous studies that failed to find the effect used a ramp, pattern $C$ in Figure 1, which induces only a small chromatic shift. Nevertheless, our results are consistent with the notion that the mechanism that underlies this small shift is the same as that which underlies the larger shifts seen in the other patterns.

\section{REFERENCES}

Chevreul, M. E. (1967). Principles of harmony and contrast of colors. New York: Van Nostrand. (Original work published 1839)

DAW, N. W. (1964). Visual response to gradients of varying colour and equal luminance. Nature, 203, 215-216.

Ercoles-Guzzoni, A. M., \& Fiorentini, A. (1958). Simultaneous contrast effect produced by non-uniform coloured fields. Atti fond Giorgio Ronchi, 13, 135-144.

FrY, G. A. (1948). Mechanisms subserving simultaneous brightness contrast. American Joumal of Optometry, 25, 162-178.

GREEN, G. A., \& FAST, M. B. (1971). On the appearance of Mach bands in gradients of varying color. Vision Research, 11, 1147-1155.

INGLING, C. R., \& DRUM, B. A. (1973). How neural adaptation changes chromaticity coordinates. Journal of the Optical Society of America, 63, 369-373.

JACOBson, J. Z., MAcKInNon, G. E. (1969). Coloured Mach bands. Canadian Joumal of Psychology, 23, 56-65.

MACH, E. (1959). The analysis of sensations. Xxxxxxxxx: Dover. (Original work published 1886)

MichaEL, C. R. (1978). Color vision mechanisms in monkey striate cortex: Dual opponent cells. Joumal of Neurophysiology, 41, 572-588.

Thouless, R. H. (1922). Some observations on contrast effects in graded discs. British Journal of Psychology, 13, 301-307.

VAN DER Horst, G. J. C., \&OUMaN, M. A. (1967). On searching for Mach band type phenomena in colour vision. Vision Research, 7, 1027-1029.

Wark, C., Cowan, W. B. (1983). The chromatic Cornsweet effect. Vision Research, 23, 1075-1077.

WILSON, H. R., \& BERGEN, J. B. (1979). A four mechanism model for threshold spatial vision. Vision Research, 19, 19-32.

WoOtBN, B. R. (1970). The effects of simultaneous and successive contrast on spactral hue. Unpublished doctoral dissertation, Brown University.

(Manuscript received November 18, 1985; revision accepted for publication September 17, 1986.) 